Peter Dippoldsmann

\title{
EG-Datenschutz - »Zwiedenken« auf europäisch Zum Gebrauch des Datenschutzes als Instrument zu seiner Vereitelung
}

Mit Datum vom I 5. 10. 1992 hat die EG-Kommission nach der Stellungnahme des Europäischen Parlaments und Stellungnahmen durch den Wirtschafts- und Sozialausschuß einen "geänderten Vorschlag für eine Richtlinie des Rates zum Schutz natürlicher Personen bei der Verarbeitung personenbezogener Daten und zum freien Datenverkehr «" vorgelegt. Dieser gegenüber dem I. Datenschutzrichtlinienvorschlag der EG-Kommission² geänderte Vorschlag (2. Datenschutzrichtlinie) ist die Basis für den vom Rat zu bildenden "gemeinsamen Standpunkt « gem. Art. 189cEG-Vertrag. Im Kern geht es damit um die Verwirklichung des informationellen Selbstbestimmungsrechts (Art. I, 2 GG) als Ausdruck des Schutzes der Menschenwürde und der Persönlichkeit und seine Umsetzung in konkrete Datenschutzregelungen in Europa. ${ }^{3}$

\section{Europäische Datenschutzrichtlinie: Erwartungen und Bedenken}

Wer glaubte, der 2. Datenschutzrichtlinienvorschlag würde gegenüber dem r. Verbesserungen im Sinne demokratischer Strukturen und eines ernstgenommenen Persönlichkeitsschutzes bringen, sieht sich getäuscht.

Am r. Datenschutzrichtlinienvorschlag war vielfach noch begrüßt worden, daß mit ihm erstmals der Versuch gemacht werde, eine einheitliche Regelung des Datenschutzes auf einem hohen Niveau für die Gemeinschaft verbindlich festzuschreiben, und es wurde auf eine Reihe positiver Einzelregelungen explizit hingewiesen ${ }^{4}$ : "Es würde somit ein Anfang gemacht, den bislang rechtsfreien Raum insbesondere bei Datenübertragungen innerhalb der Gemeinschaft ansatzweise für die von der Verarbeitung betroffenen Menschen transparent, durchschaubar und beherrschbar zu machen. «s

Grundlegende Bedenken wurden gegen die Ausrichtung des Persönlichkeitsschutzes auf den freien Datenverkehr geäußert: So solle »der hohe Schutzstandards« dem zentralen Zweck dienen, das Funktionieren des Binnenmarktes zu begleiten und zu fördern und helfen »die Hemmnisse für den Austausch von Daten abzubauen, der für das Funktionieren des Binnenmarktes unerläßlich ist« (7).

\footnotetext{
1 KOM (92) 422 endg. - SYN 287.

2 Vorschlag der EG-Kommission fur eine Richtlinie des Rates zum Schutz von Personen bet der Verarbeitung personenbezogener Daten (KOM (90) 314 endg.-SYN 287 - im folgenden I. Datenschutzrichtlinie); vgl. auch Stellungnahme zum „Vorschlag der Kommission der Europaischen Gemeinschaften fur eine Richtlinie des Rates zum Schutz von Personen bet der Verarbeitung personenbezogener Daten (KOM (90) 314 endg. - SYN 287) « der Arbeitsgemennschaft der Betriebs- und Personalrate der hochschulfreien Forschungseinrichtungen (AGBR), Arbeitskress Datenschutz, November 1991 (AGBR 199I).

3 Vgl. zum informationellen Selbstbestimmungsrecht auch auf europaischer Ebene ausfuhrlıch P. Dippoldsmann, Datenschutz und Informationsfreiheit in Eur'pa, in: Demokratie und Recht (DuR) Heft 4, 1991, S. 43 I ff. (Dippoldsmann 1991).

4 Vgl. AGBR 1991 (Fn. 2), S. if.

s AGBR i991 (Fn. 2), S. 1.
} 
Die 2. Datenschutzrichtlinie enthält wie der $\mathbf{x}$. Vorschlag Regelungen, die - isoliert betrachtet - positiv einzuschätzen sind (z. B. Wegfall der formellen Unterscheidung zwischen öffentlichem und privatem Sektor, Einbeziehung der Datenerhebung in ihren Regelungsbereich). Insgesamt und im einzelnen bestätigt sie dennoch die früher geäußerten Befürchtungen: sie handelt von einer den Binnenmarkt integrierenden Datenfluß- und -verkehrsordnung, bei der der Persönlichkeitsschutz nur Mittel zum Zweck ist. Ziel ist ein flexibel steuerbarer, reibungsloser Fluß personenbezogener Daten. Damit steht der Persönlichkeitsschutz unter dem Vorbehalt ökonomischer "Notwendigkeiten" und des Ausbaus der entsprechenden Potentiale der Informations- und Kommunikationstechniken.

Nach den offiziellen Unterlagen ist Ziel »die Errichtung und das Funktionieren des Binnenmarktes «, in dessen Rahmen »eine Harmonisierung, die den Schutz der Personen gewährleistet", angestrebt wird (z.B. S. 8). So wird an anderer Stelle zur Ermächtigung der Mitgliedsstaaten, Rechtsvorschriften zur näheren Bestimmung von Rechtmäßigkeitsvoraussetzungen der Datenverarbeitung zu erlassen, formuliert: "Sie dürfen den Grundsatz des freien Verkehrs der Daten innerhalb der Gemeinschaft nicht in Frage stellen " (S. 14). Denn: »Die Ströme personenbezogener Daten ... nehmen gerade aufgrund der Vollendung des Binnenmarktes ständig spürbar zu. Erhebliche Unterschiede «wischen den einzelstaatlichen Rechtsvorschriften schaffen "Hindernisse im freien Verkehr personenbezogener Daten" sowie "Wettbewerbsverfälschungen zwischen den Wirtschaftsbeteiligten der Gemeinschaft«. Außerdem fördert die "Einführung von Datenschutzvorschriften die Entwicklung des Informationsmarktes «?

Diese explizite Unterordnung des Persönlichkeitsschutzes als ökonomische (nicht menschenrechtlich definierte) Funktion unter den freien Fluß der Daten findet schon ihren Niederschlag im gegenüber der r. Datenschutzrichtlinie geänderten Titel, der den freien Datenverkehr explizit zum zentralen Gegenstand der Regelung macht. Was von dem so funktionalisierten Persönlichkeitsschutz real übrig bleibt, sind Überlegungen, wieviel Menschenrechtsverwirklichung der Binnenmarkt braucht, um zu funktionieren und um die Akzeptanz der BürgerInnen oder ArbeitnehmerInnen zu erhalten ${ }^{8}$.

\section{Richtlinie zum Datenschutz? - „Zwiedenken " auf europäisch!}

Grundsätzlich geht es bei dem Richtlinienentwurf um den freien Verkehr der Daten im Sinne einer sich globalisierenden kapitalistischen Ökonomie. Dieser Zweck des Richtlinienentwurfs wird aber (auf der Basis eines fiktiven "Datenschutzes«) mit dem Etikett "Schutz personenbezogener Daten « gehandelt. Entsprechend wurde von Beginn an die Kennzeichnung als »Datenschutzrichtlinienentwurf « aufgrund der bestehenden gesellschaftlichen Definitionsmacht der am freien Verkehr der Daten wirtschaftlich und politisch Interessierten zum allgemeinen Sprachgebrauch gemacht. Hinter dieser faktisch durchgesetzten Sprachregelung verschwindet der eigentliche Zweck des Richtlinienvorschlags, verschwindet die Widersprüchlichkeit der Interessenlage. Es wird der Anschein erweckt, als seien beide Interessenpole Persönlichkeitsschutz/informationelle Selbstbestimmung und freier Fluß der Daten

$6 \mathrm{~lm}$ folgenden Seitenangaben des 2. Datenschutzrıchtinienentwurfs in Klammern.

7 Folgenabschatzung zur geanderten Richtlinie S. I $25 f$.

$8 \mathrm{Vgl}$. ausfuhrlich die eınleitende Begrundung der 2. Datenschutzrichtlinie, vor Kapitel I, insbesondere Punkte $2-8$ (S. 42 ff.) und Folgenabschatzung S. 128. 
- zumindest in Einklang zu bringen. Es soll gedacht werden, daß der freie Datenverkehr etwas mit dem Persönlichkeitsschutz Identisches habe, zumindest aber miteinander vereinbar sei. Unveräußerliche, für jeden gültige (allgemeingültige) Menschenrechte werden so verhandelbar, zum Verhandlungsobjekt gemacht. Sie werden abhängig vom ökonomischen, sozialen und politischen Kräfteverhältnis, vom historisch jeweils gegebenen gesellschaftlichen Herrschaftsverhältnis zwischen den beteiligten Kontrahenten.

Diese Technik der (scheinbaren) Befriedung widersprüchlicher Interessen und der Köpfe der Menschen, die Durchsetzung dieses »Denkverfahrens «, also die Manipulation des Denkens und der Wirklichkeitswahrnehmung durch die Menschen, kennzeichnet George Orwell in seinem Roman » 1984 « $^{9}$ als totalitäre Herrschaftstechnik, »in der Neusprache als Zwiedenken bekannt “ ${ }^{\circ}$, um sich an der Herrschaft zu behaupten. Das heißt für den Bereich der Informations- und Kommunikationstechniken, sich z. B. die freie Verfügungsmacht über die Datenobjekte zu sichern.

Um von vornherein gezielten oder ungezielten Mißverständnissen oder Unterstellungen vorzubeugen: wenn hier von totalitärer Herrschaftstechnik gesprochen wird, dann meint dies "totalitäre Herrschaftstechnik « bzw. Entwicklung totalitärer Momente im gesellschaftlichen Herrschaftssystem und nicht »totalitäre Gesellschaft «. Auch in der Demokratie können sich totalitäre Herrschaftsmomente entwickeln, ohne sie damit gleich zum totalitären System zu machen. Da jedoch Gesellschaften, insbesondere demokratische, keine ein für alle Male festgefügten, unveränderlichen Blöcke sind, sondern permanenten Veränderungen in Abhängigkeit von der Entwicklung der ökonomischen, sozialen oder politischen Verhältnisse unterliegen, stellen Herrschaftstechniken zumindest einen Indikator für mögliche Entwicklungsrichtungen im (demokratischen) Herrschaftssystem dar. Totalitäre Herrschaftstechniken lassen insoweit also Schlüsse auf den Grad der Ausbildung oder des Abbaus demokratischer Verhältnisse oder Strukturen zu. Gerade in demokratischen Verhältnissen sollten aber mögliche Entwicklungen besonders aufmerksam zur Kenntnis genommen und nicht bagatellisiert werden, wie dies z. B. bei der momentanen rasanten Rechtsentwicklung in der Bundesrepublik z. B. von seiten der herrschenden Politik geschieht.

Charakter und Funktion dieses Zwiedenkens beschreibt Orwell folgendermaßen: "Die offizielle Ideologie wimmelt von Widersprüchen, auch dort, wo keine praktische Notwendigkeit für sie besteht. ... Sogar die Namen der vier Ministerien, von denen wir regiert werden, grenzen in ihrer offenen Umkehrung der Tatsachen an schamlosen Hohn. Das Friedensministerium befaßt sich mit Krieg, das Wahrheitsministerium mit Lügen, das Ministerium für Liebe mit Folterung und das Ministerium für Überfluß mit Einschränkung. Diese Widersprüche sind nicht zufällig, auch entspringen sie nicht einer gewöhnlichen Heuchelei: Es ist die wohlüberlegte Anwendung von Zwiedenken. Denn nur dadurch, daß Widersprüche miteinander in Einklang gebracht werden, läßt sich die Macht unbegrenzt behaupten.... Wenn die Gleichheit der Menschen für immer vermieden werden soll - wenn die Oberen, wie wir sie genannt haben, dauernd ihren Platz behaupten sollen -, dann muß die vorherrschende Geistesverfassung staatlich beaufsichtigter Irrsinn sein. «"

Spätestens seit der "Wende " von 1982 hat sich in der Bundesrepublik "Zwiedenken « als sprachlich vermittelte Funktion der Manipulation der Wirklichkeitswahrnehmungen und des Denkens immer offener als Instrument in der politischen Praxis

9 Vgl. George Orwell, 1984 , Ullstein 1976, z. B. S. so ff., 195 ff., 274 ff.

10 Ebd., S. 196.

I I Ebd., S. 199 f. 
entwickelt. So wurde das Beschäftigungsförderungsgesetz als „Gesetz über arbeitsrechtliche Vorschriften zur Beschäftigungsförderung «12 offiziell gekennzeichnet. Faktisch ging es aber darum, vor allem durch die systematische Einführung von Zeitverträgen, Arbeitsplatzsicherheit zu beschränken bzw. zu beseitigen. So dient das Wohnraumbeschaffungsgesetz dazu, den Mieterschutz zu unterminieren bzw. abzubauen. Jüngstes Beispiel ist der Arbeitszeitrechtsgesetz-Entwurf ${ }^{13}$ der Bundesregierung, der offiziell mit dem Etikett »Verbesserung des Gesundheitsschutzes « versehen wurde, der aber durch Ökonomisierung und entsprechende Flexibilisierungsmöglichkeiten der Arbeitszeit ohne feste Grenzziehungen und Höchstarbeitszeitbedingungen jeglichen Gesundheitsschutzgedanken zur Farce macht. Obwohl anders deklariert wird das eigentliche Ziel, eine fast grenzenlose Arbeitszeitflexibilisierung zu erreichen, konsequent auf Kosten der Gesundheit der ArbeitnehmerInnen durchgesetzt.

Die Nicht-Gewährung von Schutz oder sein Abbau wird unter dem Etikett Schutz betrieben. Dies gilt auch für den EG-Datenschutzrichtlinienentwurf: Die in Begriff und Realität des freien Datenverkehrs liegende Tendenz, jeglichem Persönlichkeitsschutz oder informationellen Selbstbestimmungsrecht die reale, objektive Grundlage zu entziehen, wird als Regelwerk des Persönlichkeitsschutzes sprachlich konstruiert, und die ihm gegenläufigen Interessen werden damit kaschiert. Mit dem Vokabular des Persönlichkeitsschutzes wird der freie Datenverkehr auch konzeptionell (4.) und im einzelnen regelungstechnisch (5.) durchgesetzt. Zwiedenken auf europäisch.

\section{Datenschutz als "selektives System "}

Im Interesse des freien Datenverkehrs konzipiert die Kommission den Datenschutz als »selektives System« (z. B. S.6). Dieses Konzept operiert durchgängig mit dem Begriff des Privatsphärenschutzes und der sensiblen oder Risiko-Daten: »... es (gibt) bestimmte Kategorien von Daten (...), die aufgrund ihres Inhalts das Risiko mit sich bringen, das Recht der betroffenen Person auf Privatsphäre zu verletzen « (S. I 8). Der Persönlichkeitsschutz soll einerseits an Verarbeitungen ausgerichtet sein, »die besondere Aufmerksamkeit erfordern, weil sie Risiken mit sich bringen«. Andererseits sollen die Verarbeitungen erleichtert oder von Auflagen (z. B. Meldepflichten, Kontrollbehörden) befreit werden, »deren Durchführung im Hinblick auf die Rechte und Freiheiten der betroffenen Personen keine Risiken birgt« (S. 6). In dieser Sicht bestehen zwischen der Verarbeitung personenbezogener Daten und den Persönlichkeitsrechten der Betroffenen erst einmal keinerlei Beziehungen. Sie kommen erst dann zustande, wenn ein irgendwie gearteter »innerer Kern der Persönlichkeit « (Privatsphäre) als berührt nachgewiesen und dies zudem noch als Risiko für das Persönlichkeitsrecht angesehen wird.

Die EG-Kommission wählt diesen Ansatz trotz ihrer formulierten Einsicht, daß nach herrschendem Verständnis davon auszugehen ist, daß die Verletzung der Persönlichkeitsrechte nicht vom Inhalt oder der Art der personenbezogenen Daten abhängig ist, sondern vom Kontext, in dem sie stehen und verarbeitet werden (Pragmatik der Datenverarbeitung). Danach gibt es also keine problematischen oder Risiko-Daten, sondern ihre Bedeutung ergibt sich immer erst aus ihrem jeweiligen Verwendungszusammenhang (S. I 8). Logische Konsequenz einer solchen Betrachtungsweise ist, daß jede Datenverarbeitung grundsätzlich eine Gefährdung bzw.

12 Vom 26. April 1985 (BGBl. S. 710 )

13 Entwurf eines "Gesetzes zur Vereinheitlichung und Flexibilisıerung des Arbeitszeitrechts (ArbZRG)" vom 5.7. 1993, Bundesrats-Drucksache (BR-Drs.) $507 / 93$. 
einen Eingriff in die Persönlichkeitsrechte der Menschen darstellt. Ein Eingriff in Menschenrechte kann ${ }^{i}$ unter demokratischen Gesichtspunkten aber nur durch gesetzliche Regelungen oder im Rahmen eines »informed consent erfolgen (Prinzip des Verbots mit Erlaubnisvorbehalt) mit der Folge größerer Transparenz (Öffentlichkeit), Einflußnahme, Berechen- und Beherrschbarkeit für die Betroffenen.

Weder die »Privatsphäre noch was als riskant oder sensibel angesehen wird oder in einem konkreten Verwendungszusammenhang dazu werden könnte, erlauben eine für die BürgerInnen/ArbeitnehmerInnen durchschau- und berechenbare Grenzziehung. Die EG-Kommission versucht auch gar nicht beides im Sinne einer operationalen Definition handhabbar zu machen. Im Gegenteil: Diese Variabilität oder Offenheit für vielfältige Interpretationen erfüllt objektiv den Zweck für die »Herren der Datenverarbeitung «, den »Persönlichkeitsschutz« im Rahmen ihrer Definitionsund Entscheidungsmacht den jeweiligen Erfordernissen des freien Datenverkehrs anpassen, ihn flexibel handhaben zu können.

\section{Prämissen und regelungstechnische Instrumente zur Sicherung des freien Datenverkebrs}

Die Ausrichtung an einem flexibel handhabbaren Persönlichkeitsschutz - der freie Datenverkehr findet seine Grenze erst bei »sensiblen «, »riskanten « Daten und an der "Privatsphäre« - hat unter dem Aspekt des Persönlichkeitsschutzes unakzeptable Konsequenzen für Einzelregelungsbereiche und ihre Prämissen bzw. für die Art der Ausgestaltung von Einzelregelungen.

\section{I Selektive Betrachtung: z. B. Datenverarbeitung für private Zwecke}

In der Richtlinie wird erst gar nicht versucht, die Verarbeitung für "ausschließlich private Zwecke « unter dem Aspekt des heutigen Stands der Technik zu problematisieren. Es wird nicht hinterfragt, ob eine solche Abgrenzung überhaupt noch den gegebenen, erst Recht den zukünftigen Realitäten entspricht/entsprechen kann. Sie wird schlicht mit dem Hinweis auf ihre Harmlosigkeit - Stichwort: "Notizbuch « aus dem Anwendungsbereich der Richtlinie ausgenommenen. Gerade wegen der immer leistungsfähigeren Verarbeitungstechnik, immer vielfältigeren PC-Anwendungen in allen Bereichen der Gesellschaft, des zunehmenden Ausbaus der Netzstrukturen, der Auslagerung und Privatisierung von informationellen Arbeiten/Dienstleistungen aus Unternehmen und Verwaltungen werden die Grenzen zwischen privaten, gesellschaftlichen oder ökonomischen Zwecken (Werkverträge, Heim-(Tele-)arbeit) technisch-organisatorisch zunehmend verwischt. Die offensichtlichen Unterschiede schon in der Gegenständlichkeit beider Informationsträger werden ebensowenig zur Kenntnis genommen wie die Erkenntnisse, die in der Diskussion um Datenschutz und Datensicherung herausgearbeitet wurden (Probleme des online-Zugriffs, der Datenübermittlung, der Schrankenziehung für die Vernetzung von Computern). Deshalb ist es zumindest naiv (wenn man nicht »wider besseres Wissen « unterstellen will), zu behaupten, ein privates elektronisches sei einem traditionellen Notiz- oder Adreßbuch vergleichbar und bedürfe keiner Regelung. 
Ähnlich bedenkenlos wird in der Richtlinie mit der Werbung (Verbraucherschutz) umgegangen, was insbesondere ArbeitnehmerInnen in ihrer Rolle als KonsumentInnen betrifft. Hier haben sich eindeutig die Interessen der Werbe- und Adressenhandelsbranche durchgesetzt. Um diesen Interessen gerecht zu werden, wählt die Kommission folgendes Konstrukt: Werbung wird schlicht als gegeben vorausgesetzt und gar nicht erst hinterfragt, obwohl der manipulative und die "Privatsphäre" beeinträchtigende Charakter von Werbung vor allem unter den heute gegebenen Bedingungen der informations- und kommunikationstechnischen Möglichkeiten (z. B. Konsumentenprofil) auch der Kommission bekannt sein muß. Werbung wird so zur harmlosen Veranstaltung, die die Werbeobjekte nicht beeinträchtigen kann (kein Risiko). Zwar sollen »vor der Übermittlung personenbezogener Daten oder deren Benutzung im Auftrag Dritter für schriftliche Werbezwecke (was immer dies auch heißen mag) die betroffenen Peronen ausdrücklich auf die Möglichkeit hingewiesen werden, ihre Daten ohne Kosten löschen lassen zu können « (Art. I s). Wie ernst es der Richtlinienvorschlag aber mit dieser Information über die Löschungsmöglichkeit wirklich meint, macht die Begründung zu diesem Artikel überdeutlich: »Der Verantwortliche der Verarbeitung kann seinen Verpflichtungen bei seinen regelmäßigen Kontakten mit den betroffenen Personen nachkommen, ohne notwendigerweise eine spezifische Korrespondenz zu führen (S.26). Eine solche Regelung macht ein vages, zufälliges und unkalkulierbares Informationsverfahren zur Voraussetzung der Zulässigkeit von Werbung. Es bleibt auch unklar, ob Art. Is Abs. 1 - ein Widerspruch kann nur aus »berechtigten Gründen « eingelegt werden - auch auf den Löschungsanspruch angewandt werden wird. Dies alles ist für einen ausreichenden Persönlichkeitsschutz ungeeignet. Die Barriere gegen einen für die Menschen unkontrollierbaren und undurchschaubaren, für die entsprechenden Kapitale freien Datenverkehr wird auf den denkbar niedrigsten Punkt heruntergeschraubt.

\subsection{Der stumme Zwang der Verbältnisse: z. B. ArbeitnebmerInnendatenschutz}

Die 2. Richtlinie schafft mit ihrer Konzeption und deren Umsetzung in Einzelregelungen Rahmenbedingungen für einen ArbeitnehmerInnendatenschutz, der einen solchen Namen nicht mehr verdient. Gerade die Orientierung auf den freien Fluß der Daten hat zur Konsequenz, daß noch die letzten Barrieren z. B. zwischen international operierenden Unternehmen einerseits, zwischen ihnen und den staatlichen Bürokratien, insbesondere den Sozialbürokratien und ihren undurchschaubaren Sozialinformationssystemen andererseits beseitigt werden. Dem Persönlichkeitsschutz der BürgerInnen in ihrer Rolle als abhängige ArbeitnehmerInnen wird so von vorneherein die Grundlage entzogen. Denn trotz der fiktiven und formalen Vertragsfreiheit befinden sich ArbeitnehmerInnen in einer existentiellen Zwangssituation, einem sozialen Abhängigkeitsverhältnis, insbesondere unter den Bedingungen einer hohen und steigenden Arbeitslosigkeit, die sie schon aufgrund des "stummen Zwangs der Verhältnisse « erpreßbar macht. Dies wird zwar in der 2. Richtlinie ansatzweise zugestanden. Die Konsequenzen aus dieser Einsicht werden jedoch nicht in klare Regelungen umgesetzt. 
Grundsätzlich formulierte Einsichten in Abhängigkeitsverhältnisse und Prinzipien (z. B. Einwilligung) werden zwar benannt, dann aber aufgeweicht und/oder weitgehend zurückgenommen. Regelungstechnisch wird dies z.B. folgendermaßen gehandhabt:

* Der zuerst formulierte Grundsatz der Einwilligung wird nachfolgend als eine Zulässigkeitsvoraussetzung der Datenverarbeitung unter vielen anderen relativiert. Trotz Betonung ihrer Bedeutung ist sie dann gerade nicht mehr entscheidende Grundlage der Datenverarbeitung. Die freiwillige, informierte Zustimmung zur Datenverarbeitung (»informed consent «) ist ihres prinzipiellen Charakters entkleidet.

* Von dem juristischen Regel-Ausnahme-Prinzip wird so extensiv Gebrauch gemacht, daß die Regel kaum noch erkennbar ist (z. B. Rechte der Betroffenen, Meldepflichten).

* Sehr vage, interpretationsfähige Begriffe wie "öffentliche Sicherheit «, »wichtiges öffentliches Interesse«, »vernünftigerweise«, »berechtigte Interessen" usw. und willkürliche Wertungen, was als nicht riskant einzuschätzen ist, ergänzen dieses Bild.

* Hinzu kommen "Klarstellungen« zur Frage der Abhängigkeitsverhältnisse, die allenfalls als gutgemeinter Appell unter Vermeidung normativer bzw. regelungstechnischer Konsequenzen gewertet werden können: „Die Einwilligung (hat) in den Fällen, in denen möglicherweise Druck auf die betroffene Person ausgeübt wird « (Fall des Arbeitnehmers gegenüber seinem Arbeitgeber...), »ohne Zwang « zu erfolgen ( 11 , Art. $2 \mathrm{~g}$ ).

Wäre mit der Richtlinie ernsthaft der Schutz sozialer Abhängigkeitsverhältnisse intendiert, dann hätten - entsprechend der eigenen Grundkonzeption (»Risikobewertung «/besondere Gefährdung der ArbeitnehmerInnen) - klare Grenzen für die Datenverarbeitung etwa für das Arbeitsverhältnis gezogen werden müssen. Abgestufte Schutzinstrumente sind z. B.:

* Datenverarbeitungsverbote,

* Verbote mit Erlaubnisvorbehalt (z. B. strikte schriftliche Einwilligung, »informed consent «, klare Sanktionsmöglichkeiten),

* insgesamt also enge Grenzziehungen im Hinblick auf Zulässigkeit und Erforderlichkeit von Datensammlungen und -verarbeitungen (z. B. Zwang zu Datensparsamkeitsprüfungen, Wahl weniger eingreifender Alternativen),

* klare, eindeutige Begriffsbildungen und

* verstärkte Öffentlichkeits-, Einfluß- und Kontrollverfahren.

Diese Instrumente dürfen nicht isoliert voneinander, sondern sie müssen ergänzend, sich in ihren Wirkungen gegeneinander flankierend bzw. absichernd gesehen werden.

In der Richtlinie wird jedoch noch nicht einmal der Weg eingegrenzter, eindeutiger Voraussetzungen für die Rechtmäßigkeit der Verarbeitungen eingeschlagen. Sie ist geradezu bemüht, sie möglichst offen zu halten. Wie vorher schon gesagt, soll die Einwilligung nicht mehr - im Gegensatz zum I. Richtlinienvorschlag - »als wichtigstes Kriterium angesehen (werden), von dem es Ausnahmen gibt, sondern als eine von mehreren möglichen Voraussetzungen « (S. 17, Art. 7). Es soll der Eindruck vermieden werden, daß »für jede Verarbeitung die zuvorige Einwilligung der betroffenen Person erforderlich sei, während es sich nur um eine der Grundlagen der Zulässigkeit der Verarbeitung handelt. « 
Die Bestimmungen über die Einwilligung werden in ihrer eigentlichen, grenzziehenden Bedeutung zugunsten der Einflußnahme der Betroffenen auch dadurch relativiert, daß sie in »die Form einer Definition « (S. 1 r) zurückgestutzt werden. Sie wurden auch dahingehend abgeschwächt, daß "der Bezug auf den ausdrücklichen Charakter der Einwilligung... gestrichen (wurde), um zu verhindern, daß dies als Erfordernis einer schriftlichen Erklärung ausgelegt werden kann « (S. 11). Den üblichen Unterstellungen (Definitionsmacht), Betroffene hätten ihre Einwilligung deutlich gemacht, ist damit zukünftig Tor und Tür geöffnet.

\subsection{Entgrenzungen: z. B. Zulässigkeit der Datenverarbeitung}

Hinzu kommen die weiteren Kriterien für eine zulässige Datenverarbeitung, mit denen das Verfahren noch offener, flexibler gestaltet wird. Sie schließen faktisch keine halbwegs bedeutsame Verarbeitung mehr aus - und sei sie für die Betroffenen noch so »riskant «. Die Verarbeitung personenbezogener Daten soll stets dann zulässig sein, wenn sie zur »Erfüllung eines Vertrages erforderlich« ist. Damit bleibt - wie auch hier die praktische Erfahrung im Arbeitsverhältnis zeigt - der Persönlichkeitsschutz grundsätzlich von den faktischen sozialen Machtverhältnissen abhängig. Erweitert wird der Vorschlag um

" die herrschaftlich-fürsorgliche Zulässigkeitsvoraussetzung der "Erforderlichkeit für die Wahrnehmung lebenswichtiger Interessen der betroffenen Person « (welche sind das und wer entscheidet, wann dieser Fall vorliegt?),

* die "Wahrnehmung einer Aufgabe (?) in öffentlichem Interesse (?) (nicht die Wahrnehmung einer öffentlichen Aufgabe) oder "in Ausübung der Staatsgewalt» sowie

* „die Wahrnehmung des Allgemeininteresses" oder "berechtigter Interessen" von Außenstehenden (?), "sofern nicht das Interesse der betroffenen Person überwiegt « (Art. 7).

Die Richtlinie schafft auch auf diese Weise die Grundvoraussetzungen für eine wirklich freie Datenverarbeitung, bei der extreme Vorfälle als Randkorrekturen unter das Schild »Persönlichkeitsschutz« eingeordnet werden dürfen.

Aufgrund der weiten Fassung, der großzügigen Zulassungskriterien bei der Verarbeitung personenbezogener Daten (Art. 7) bleibt bei diesem "Herzstück « des 2. Richtlinienvorschlags nichts wesentliches vom behaupteten Persönlichkeitsschutz übrig. Dies gilt insbesondere für Abhängigkeitsverhältnisse. Eine Regelung im Sinne des Persönlichkeitsschutzes müßte grundsätzlich anders gestaltet werden. Die Zulässigkeitsvoraussetzungen müßten eng eingegrenzt werden und zumindest unter dem Verbot mit Erlaubnisvorbehalt stehen.

\subsection{Der Verweis auf den Verweis: z. B. Zweckbindung, Übermittlung, Online-Verfabren}

Das Verfahren der Richtlinie, wohlklingende Grundsätze zu formulieren und sie im gleichen Atemzug wieder zurückzunehmen, findet sich auch hierbei wieder. So müssen zwar »die Daten... für bestimmte, ausdrücklich festgelegte und rechtmäßige Zwecke erhoben« werden, verwendet werden dürfen sie jedoch auch dann, wenn sie »in einer mit diesen $Z$ weckbestimmungen zu vereinbarenden Weise« verarbeitet werden (Art. 6 Abs. I b). Eine strikte Zweckbindung wird so von vornherein aufgegeben. Die Daten dürfen auch bei späterer $Z$ weckänderung verwendet werden (S. 1 5). Sie brauchen für diese schon aufgeweichte Zweckbestimmung nicht mehr erforderlich oder für den Zweck geeignet sein. Es reicht, daß sie »den Zwecken entspre- 
chen (?)..., dafür erheblich (?) (sind) und nicht darüber hinausgehen « (Art. 6 Abs. I c). Sie müssen nicht »auf dem neuesten Stand sein «, sondern nur, "falls erforderlich" (Art. 6 Abs. I d).

Diese unkalkulierbare, aufgeweichte Zweckbindung der Datenverarbeitung ermöglicht keine klare Grenzziehung. Dies hat Konsequenzen für die Frage der Datenübermittlung, damit für Online-Verfahren und den grenzüberschreitenden Datenexport in Drittländer. So betont die Kommission: "A uf die Verarbeitung von Daten zu einem anderen Zweck als dem, zu dem die Verarbeitung ursprünglich vorgenommen wurde ... sowie auf die rechtmäßigen Kriterien für die Übermittlung personenbezogener Daten... wird nicht speziell eingegangen. Der allgemeine Grundsatz der Zweckbestimmung (geänderter Artikel 6.r.b) und die in dem geänderten Artikel 7 (Zulässigkeitsvoraussetzungen, d. V.) festgelegten Voraussetzungen werden für ausreichend gehalten « (S. I7). Zum Online-Verfahren wird dann wieder auf das »Konzept der Übermittlung« verwiesen: "Auf die >on line-Abfrage « wird nicht eingegangen, da diese im Konzept der Übermittlung enthalten ist " (S. 22). Um die Verwirrung zur Übermittlung dann vollständig zu machen, formuliert die Kommission: "Das Kriterium der Datenübermittlung an Dritte, das in dem ursprünglichen Vorschlag zugrundegelegt wurde, um die (Melde-)Verpflichtung zu begründen, wurde wegen der Kritik an seinem unangemessenen Charakter nicht aufgenommen, da bestimmte (?) Übermittlungen an Dritte die Rechte der Personen nicht beeinträchtigen« (S. 30).

Dieser regelungstechnische Umgang mit dem Datenfluß signalisiert noch nicht einmal den Versuch, wenigstens den Stand der Technik im Hinblick auf die OnlineVerfahren und der systematisch vorangetriebenen technisch-organisatorischen Vernetzung der Datenverarbeitung in die Überlegungen mit einzubeziehen.

\subsection{Was ist angemessen? z. B. Datenexport in Drittländer}

Mit »dem vorliegenden Richtlinienvorschlag« soll der freie Verkehr der Daten zwischen den Mitgliedstaaten eingeführt werden. Deshalb braucht es »für die Weitergabe in Drittländer gemeinsame Bestimmungen « ( $\mathrm{S} .35$ ); grenzüberschreitende Bewegungen personenbezogener Daten sind für die Entwicklung des internationalen Handels erforderlich « (Begründung S. 56, Nr. 25). Der Datenexport in Drittländer (Art. 26, 27) soll deshalb grundsätzlich ermöglicht werden, wenn dieses Drittland ein "angemessenes Schutzniveau « gewährleistet. Ausnahmen hiervon sind im Einzelfall möglich, wenn der Betroffene einwilligt oder der Datenimporteur auf andere Weise, etwa durch vertragliche Zusicherung von Schutzrechten, dieses Niveau sicherstellt (Verhandlungsmodell). Zusätzlich bleibt die Möglichkeit, daß »die Wahrung des öffentlichen Interesses den Transfer erforderlich macht« (Begründung S. 57, Nr. 26). Die Zulässigkeit der Übermittlung wird also nicht von gleichwertigen Datenschutzvorschriften im Empfängerland abhängig gemacht. Der Vorschlag gibt sich mit »angemessenen Vorkehrungen« zufrieden. Es werden offensichtlich geringere Anforderungen an Drittländer als im Binnenmarkt gestellt, obwohl dieser Datenverkehr für die Betroffenen noch »riskanter«, noch undurchschaubarer und unbeherrschbarer ist und die Kommission nach ihrer eigenen Risikokonzeption hier besonders hohe Anforderungen stellen müßte. Ein Grund für dieses Vorgehen liegt im Konzept des »selektiven Persönlichkeitsschutzes« im Interesse des freien Datenverkehrs begründet. 
Selbst da noch, wo die Kommission vorgibt, besonders einschränkend vorzugehen, bei den »Datenverarbeitungsverboten « besonders sensibler Daten über rassische und ethnische Herkunft, politische Meinung, religiöse, philosophische oder moralische Überzeugung oder über die Gewerkschaftszugehörigkeit, bleibt es nicht beim Verbot. ${ }^{14}$ Obwohl die Persönlichkeitsrechte in diesen Fällen nach ihrem eigenen Risikoverständnis besonders zu schützen sind, nimmt sie sie im gleichen Atemzug wieder zurück.

Ohne die Problematik der Einwilligung, insbesondere in Abhängigkeitsverhältnissen auch nur anzusprechen, soll eine schriftliche Einwilligung die Verarbeitung dieser Daten zulässig machen. "Eine politisch, philosophisch, religiös oder gewerkschaftlich ausgerichtete Stiftung oder Vereinigung « ohne Erwerbszweck darf unter bestimmten Voraussetzungen (z. B. »im Rahmen ihres berechtigten Ziels «) diese Daten verarbeiten. Und es bleibt die alles ermöglichende Formel: Die Verarbeitung dieser Daten kann erfolgen, wenn »die Verarbeitung... unter solchen Bedingungen (erfolgt), daß sie die Privatsphäre und die Grundfreiheiten offenkundig nicht beeinträchtigt « (Art. 8 Abs. 2 a-c).

Selbst wenn man aber einmal unterstellt, es handele sich hierbei um den ernsthaften Versuch eines wirksamen Verbotes, dann bleibt immer noch der Tatbestand, daß diese Verbote in der Realität explizit ihrer tatsächlichen Grundlage beraubt werden. Die Sozial- und Gesundheitspolitik der EG ist gerade auf die restlose Erfassung eines Teils dieser Daten ausgerichtet's. Im Programm »Einsatz der Informations- und Kommunikationstechnologie im Sozial- und Gesundheitswesen - AIM (Fortgeschrittene Informationsverarbeitung in der Medizin - ABL N. L 3 I4/22) k heißt es deshalb: »AIM wird einen wichtigen Beitrag zur Schaffung eines integrierten Gesundheitsumfelds (IHE) darstellen. Dies bedeutet, daß sämtliche Formen der Gesundheitsfürsorge erfaßt werden, angefangen bei der Vorsorge über die primäre bis hin zur sekundären Versorgung $\ll(\text { S. } 28)^{16}$.

Auch unter diesem Gesichtspunkt der Weiterentwicklung der realen und technischen Verhältnisse ist die Datenschutzrichtlinie allenfalls als Akzeptanz fördernde Maßnahme zu beurteilen. Ein Persönlichkeitsschutz ist ohne ihre grundlegende Änderung und ohne echte Verarbeitungsverbote (z. B. Verbot der Genomanalyse im Arbeitsverhältnis ${ }^{17}$, Abschaffung von sog. Sicherheitsüberprüfungen) nicht zu gewährleisten.

14 "Die Mitgliedstaaten untersagen die Verarbeitung von Daten, aus denen die rassische und ethnische Herkunft, die politische Meinung, die religiose, phılosophische oder moralische Uberzeugung oder die Gewerkschaftszugehorıgkeit hervorgehen, sowe von Daten uber die Gesundheit und Sexualleben* (Art. 8 Abs. I).

is Vgl. o.A., Medizinische Daten besser verarbeiten, in: Woche im Bundestag (wib) 1992, r1. I1. I992, S. I 8 .

16 Vgl. P. Dippoldsmann, Informations- und Kommunikationstechniken im Sozial- und Gesundheitssystem - einzelstaatliche und europaische Entwicklungen, in: FIFF-Kommunıkatıon, Heft 4, 1993, 5. 23 ff; vgl auch Th. Weichert. Lediglich der Akzeptanztrottel einer begehrlichen Verwaltung. Zehn Jahre Grundrecht auf Datenschutz - Kein Grund zum Feiern, in: Frankfurter Rundschau, 29. 12. 1993, S. I6.

17 Vgl. Stellungnahme der Konferenz der Arbeitsgemeinschaft der Betriebs- und Personalrate der hochschulfreien Forschungseinrichtungen (AGBR) zur Genomanalyse im Arbeitsverhaltnis, Einstellungskriterium DNA? ForscherInnen gegen betrieblıche Genomanalyse, in: Forum Wissenschaft, Heft 2, 1993, S. 44 ff. (AGBR I993). 
5.9 Zwingende Staats-(sicherheits-)interessen: $z$. B. individuelle Rechte, personenbezogene Daten, Meldepflichten, -register

Soweit den Betroffenen Informations-, Auskunfts- oder Einsichtsrechte zugestanden werden, sind diese als individuelle Rechte ausgestaltet (Art. 10-14). Kollektive Kontroll- und Gestaltungsrechte sind schon gar nicht vorgesehen. Aber selbst die individuellen Rechte werden weitgehenden Beschränkungen (insbesondere Art. I4) unterworfen: Gründe zur Einschränkung sind die Sicherheit des Staates, die Landesverteidigung, Strafverfolgung und öffentliche Sicherheit. Hinzu kommen solche, in keiner Weise mehr justiziablen Kriterien wie die »ordnungsgemäß begründeten, zwingenden wirtschaftlichen und finanziellen Interessen eines Mitgliedstaates oder der Europäischen Gemeinschaft « sowie »Kontroll- oder Überwachungsaufgaben, die mit der Ausübung öffentlicher Gewalt verbunden sind oder von dem Zusammenwirken mit solchen Aufgaben herrühren.«

Indirekt wird in Art. I 4 Abs. 3 zusätzlich eine nicht unwesentliche, eingrenzende Definition des "Personenbezugs « von Daten vorgenommen. Wurde im r. Richtlinienvorschlag noch davon ausgegangen, daß Daten dann nicht personenbezogen, sie also anonym sind, wenn sie nur mit großem Aufwand an Arbeitskraft, Kosten und Zeit der Person zugeordnet werden können, soll es jetzt ausreichen, "daß die betroffenen Personen vernünftigerweise nicht bestimmbar sind (Art. 2). Damit wird aber der Begriff des "personenbezogenen Datums « eingeengt, weniger Daten fallen wegen ihrer Anonymität in den Schutzbereich der Richtlinie.

Auch die Meldepflichten (Art. $18 \mathrm{ff}$.) »für bestimmte Kategorien von Verarbeitungen werden wesentlich eingeschränkt. Damit werden die Kontrollmöglichkeiten der Betroffenen beschnitten. Dies wirkt sich insbesondere im Arbeitsbereich aus. Die Meldepflicht soll vereinfacht werden oder ganz entfallen, wenn »die Rechte und Freiheiten der betroffenen Personen nicht « beeinträchtigt werden. "Diese Verarbeitungskategorien betreffen insbesondere das Erstellen beruflicher Schreiben oder Dokumente, die Erfüllung gesetzlicher, buchhaltungsbezogener, steuerlicher oder sozialer Verpflichtungen « (Art. I 9). Was fällt dann aber noch unter die Meldepflicht? Zusätzlich wird das Einsichtsrecht der Betroffenen in das Melderegister gem. Art. 14 eingeschränkt. Insoweit gilt das oben zu den individuellen Rechten Gesagte.

\section{Zusammenfassung}

Die jetzt vorgelegte 2. Datenschutzrichtlinie ist eine Datenverkehrsordnung, die die Rahmenbedingungen für den freien Fluß der Daten schafft. Sie erfüllt in keiner Weise die Mindestanforderungen, die an eine Datenschutzregelung materiell und unter demokratischen Gesichtspunkten zu stellen sind. Sie schafft weder Transparenz noch Berechen- oder Beherrschbarkeit für die Betroffenen. Was ihnen allenfalls bleibt und nachträglich kaum hilft, ist das Entdeckungsverfahren Rechtsprechung, und das nur mit unsicherem Ausgang.

Der Richtlinienvorschlag berücksichtigt einzig die Interessen der am Verfahren beteiligten privaten und staatlichen Interessengruppen sowie Wirtschaftsinteressen. Das »Verzeichnis der Organisationen, die zu diesem Vorschlag gehört wurden, und die wesentlichen Punkte ihrer Stellungnahmen « (S. I 28$)^{18}$ geben in dieser Hinsicht ein beredtes Bild, um was und wen es bei dieser europäischen Regelung geht. Jedenfalls geht es nicht um die Interessen von BürgerInnen, ArbeitnehmerInnen oder

I8 Z. B. "europaische Berufsverbande "(?), Vereınigung der Banken in der EG, CELD, FEWITA, GEDIS, der Europaische Direktmarketingverband, EAT, CHANGE (Vereinigungen ohne Erwerbszweck), die Europaische Gemeınschaft fur Meinungs- und Marketıng-Forschung ESOMAR usw. 
Verbrauchern an der Sicherung ihrer Persönlichkeitsrechte. Zwar wird Persönlichkeitsschutz gesagt. Entwickelt werden aber die regulativen Bedingungen für die Durchsetzung des freien Datenverkehrs im Interesse einer globalen kapitalistischen Ökonomie. Der Vorschlag symbolisiert Zwiedenken auf europäisch als ein Indikator für eine Entwicklung undemokratischer Herrschaftsstrukturen.

Zur freien Entfaltung der Persönlichkeit und der Menschenwürde in demokratischen Strukturen und Verhältnissen, auch auf europäischer Ebene, liegen umfangreiche Forderungen/Forderungspakete seit langem auf dem Tisch. ${ }^{19}$ Trotz oder gerade wegen dieser Forderungen und ihrer demokratischen Entwicklungsperspektive ist gerade von den Interessenvertretern aus Ökonomie und Politik keine Vertretung zu erwarten. Ihre Durchsetzung müssen wir als BürgerInnen, ArbeitnehmerInnen oder VerbraucherInnen selbst in die Hand nehmen - im alltäglichen Leben ebenso wie auf der Ebene der öffentlichen Auseinandersetzung, innerhalb der Betriebe oder der Gewerkschaften ebenso wie auf politischer Ebene. Wer sollte es tun, wenn nicht wir selbst. 